\title{
Longitudinal MRI volumetric evaluation in patients with familial mesial temporal lobe epilepsy
}

\section{Livia Conz', Marcia Elisabete Morita ', Ana Carolina Coan', Eliane Kobayashi' ${ }^{2}$, Clarissa Lin Yasuda' , Amanda Regio Pereira ${ }^{1}$, Iscia Lopes-Cendes ${ }^{3}$ and Fernando Cendes ${ }^{1 *}$}

1 Department of Neurology, University of Campinas, Campinas, São Paulo, Brazil

2 Department of Neurology and Neurosurgery, McGill University, Montreal, QC, Canada

${ }^{3}$ Department of Medical Genetics, University of Campinas, Campinas, São Paulo, Brazil

\section{Edited by:}

Jorge G. Burneo, University of Western

Ontario, Canada

Reviewed by:

Sandrine Deribaupierre, University of Western Ontario, Canada

Paolo Federico, University of Calgary,

Canada

Seyed Mirsattari, University of Western Ontario, Canada

\section{*Correspondence:}

Fernando Cendes, Department of Neurology, University of Campinas, Cidade Universitaria, Campinas, São

Paulo 13083-970, Brazil.

e-mail: fcendes@unicamp.br
Introduction: Studies have shown progressive cerebral damage in patients with refractory mesial temporal lobe epilepsy (MTLE). However, this has not been demonstrated in benign forms of MTLE such as familial mesial temporal lobe epilepsy (FMTLE). Purpose: To evaluate progression of hippocampal atrophy (HA) in patients with sporadic mesial temporal lobe epilepsy (SMTLE) and FMTLE by longitudinal Magnetic resonance images (MRIs) acquired with at least 7 months of interval. Method:We included 35 patients with FMTLE (25 classified as benign and 10 refractory) and 33 with SMTLE (4 benign and 29 refractory). All MRIs were analyzed by an investigator blind for clinical data. Hippocampal analyses were performed manually in coronal $3 \mathrm{~mm}$ thick T1 inversion recovery, using the software Scion Image ${ }^{\circledR}$. Volumes were compared to those from a control group, and HA was determined for volumes below two standard deviations from the mean of controls. Results: The mean interval between the first (MRI1) and second MRI (MRI2) was 90 months for FMTLE and 45 months for SMTLE group. FMTLE group: volumetry demonstrated HA in 20 patients in MRI1 and in 23 patients in MRI2. There was significant progression of HA in FMTLE patients between MRIs in both benign and refractory FMTLE patients (benign FMTLE: right hippocampus, $p=0.001$ and left hippocampus, $p<0.001$; refractory FMTLE: right hippocampus, $p=0.022$ and left hippocampus, $p<0.010)$. SMTLE group: volumetry demonstrated HA in 27 patients in MRI1 and in 29 patients in MRI2. In the group analysis, there was a significant reduction of the right $(p<0.0001)$ and left $(p<0.0001)$ hippocampal volumes during the follow-up period. Although the mean time between the MRIs in the FMTLE group was twice the time of the SMTLE group, the progression of volume loss was similar in both groups, indicating a slower progression in the FMTLE patients. Conclusion: FMTLE patients have progressive hippocampal volume reduction independently of seizure frequency although the progression of HA seems to be slower than in SMTLE.

Keywords: epilepsy, mesial temporal lobe epilepsy, magnetic resonance imaging, familial temporal lobe epilepsy, hippocampal sclerosis, seizures

\section{INTRODUCTION}

Mesial temporal lobe epilepsy (MTLE) is the most common type of partial epilepsy, and it is frequently associated with hippocampal sclerosis (HS; Gastaut et al., 1975; Bruton, 1988). Magnetic resonance images (MRI) signs of HS include hippocampal atrophy (HA), abnormal shape, loss of internal structure, and T2 hyperintense signal (Cascino et al., 1991; Cendes et al., 1993; Jackson et al., 1993).

The familial form of mesial temporal lobe epilepsy (FMTLE) was first described as a benign form of partial epilepsy (Berkovic et al., 1996; Kobayashi et al., 2001). Previous studies have demonstrated the presence of MRI signs of HS among patients with FMTLE and in some asymptomatic relatives (Kobayashi et al., 2001, 2002, 2003).

Clinical and experimental studies have tried to demonstrate the progression of hippocampal damage in patients with epilepsy and its association with refractory seizures with discrepant results (Kalviainen et al., 1998; Briellmann et al., 2002; Fuerst et al., 2003; Cendes, 2005; Cendes et al., 2005). However, there are no previous studies trying to demonstrate this association in patients with FMTLE.
The objective of this study was to investigate MRI volumetric abnormalities of hippocampus of patients with FMTLE and evaluate the possibility of progression of these abnormalities in patients with FMTLE and sporadic mesial temporal lobe epilepsy (SMTLE) in a longitudinal study with a prolonged follow-up.

\section{MATERIALS AND METHODS}

Patients from our epilepsy clinic with clinical and electroencephalographic diagnosis of MTLE and at least two MRIs obtained from October 1998 and January 2008 were followed prospectively and were included in this study.

All patients were interviewed in appointments every 3-6 months during the follow-up-period and seizure characteristics were constantly accessed. Patients were only included if they had seizures with typical mesial temporal lobe semiology and no atypical findings on the EEG.

According to clinical characteristics, patients were divided in two subgroups: (1) FMTLE (patients with at least two first or second degree relatives with MTLE as defined previously Kobayashi et al., 2001) and (2) SFMTLE (patients with no family recurrence). 
Control group was composed of 14 health individuals, with no familial history of epilepsy and with two MRI performed with a minimum interval of 7 months.

Patients were also classified as benign or refractory based on the number of seizures presented on the year before the first MRI and in between the acquisition of both MRIs. Patients who had seizure remission, were seizure-free under AED or had sporadic seizures (three or less complex partial seizures per year) or had only simple partial seizures (typical MTLE auras) were considered as benign (for more detail, see Kobayashi et al., 2001).

Each patient had at least three routine EEG recordings with $30 \mathrm{~min}$ duration each, using the 10-20 electrode placement with additional temporal (T1, T2, and zygomatic electrodes) in an 18 or 32-channel digital EEG recording system, except for four patients with FMTLE who were under remission and refused to have follow-up EEGs. All patients with refractory seizures had abnormal EEG, showing epileptiform discharges over the anterior-mid temporal lobe region(s) coincident with the side of MRI showing signs of HS. In the FMTLE group, five patients who were under remission and one with rare seizures had normal routine EEGs (Kobayashi et al., 2001). The remaining FMTLE patients had abnormal EEG in one or both anterior-mid temporal lobe regions. All patients had typical history and semiology of mesial TLE (Cendes et al., 2005).

All patients signed an informed consent approved by the Ethic Committee of our institution before each MRI. Clinical data were collected prospectively. MRIs were performed in a 2T-scanner, with $\mathrm{T} 1$ and $\mathrm{T} 2$ acquisitions in three orthogonal planes. We used $3 \mathrm{~mm}$ T1- "inversion recovery" (T1-IR) coronal slices (flip angle $=200^{\circ}$; $\mathrm{TR}=2800, \mathrm{TE}=14$, inversion time $=840$, matrix $130 \times 256$, $\mathrm{FOV}=16 \mathrm{~cm} \times 18 \mathrm{~cm}$ ) for volumetric analysis.

Hippocampal volumes were determined manually (Watson et al., 1997 ) in the 3-mm T1-IR coronal images using Scion Image ${ }^{\circledR}$ software. The investigator who performed the volumetric and signal evaluation was blinded about patients' clinical data at the moment of MRI study. Hippocampal volumes were corrected by total intracranial volume for each patient to eliminate variation of the size of the brain (Watson et al., 1997). HA was determined for either total volumes or asymmetry index (smaller/larger ratio) below two standard deviations from the mean of the control group $(Z$-score $<$ or $=-2)$.

Statistical analysis was obtained with Systat $9^{\circledR}$ software. Chisquare test was used to determined differences of frequencies and paired $t$-test was used to compare the results between MRI1 and MRI2. The statistical significance was set to $p<0.05$.

\section{RESULTS}

\section{DEMOGRAPHIC ASPECTS}

\section{Familial mesial temporal lobe epilepsy}

Thirty-five patients with FMTLE were included ( 9 men; mean age 41 years, minimum 17, and maximum 71). The mean interval between MRI1 and MRI2 was 90 months (ranging from 20 to 121 months). Twenty-five patients (71\%) were classified as benign.

\section{Sporadic mesial temporal lobe epilepsy}

Thirty-three patients were included ( 14 men; mean age 36 years range from 21 to 70). The mean interval between MRI1 and MRI2 was 45 months (range from 7 to 85 months). Only four patients (12\%) were classified as benign.
There was no difference of sex distribution or age between FMTLE and SMTLE individuals or between patients and controls.

\section{VOLUMETRIC STUDY}

\section{Familial mesial temporal lobe epilepsy}

Volumetric study demonstrated HA in 20 (57\%) patients (6 right, 9 left, 5 bilateral) in MRI1 and in $23(66 \%)$ patients (7 right, 11 left, 5 bilateral) in MRI2. Group analysis demonstrated a significant difference between the volumes of patients and controls for both the first and second MRI ( $t$-test, MR1: right hippocampus, $p=0.002$; left hippocampus, $p=0.003$; AI, $p=0.017$. MR2: right hippocampus, $p<0.001$; left hippocampus, $p<0.001$; AI, $p=0.010$; Figure 1).

There was significant hippocampal volume reduction in the MRI2 when compared to MRI1 (paired $t$-test right hippocampus, $p<0.001$; left hippocampus, $p<0.001$; Figure 2).

When we divided the FMTLE patients in benign (25 (71\%) individuals) and refractory (10 individuals), it was still possible to observe significant hippocampal volume reduction in the MRI2 when compared to MRI1 in both groups (paired $t$-test, benign FMTLE: right hippocampus, $p=0.001$ and left hippocampus, $p<0.001$; refractory FMTLE: right hippocampus, $p=0.022$ and left hippocampus, $p<0.010)$. In the group of benign FMTLE, 14 patients $(56 \%)$ had HA in MR1 and $16(64 \%)$ in MRI2 and in the refractory group $6(60 \%)$ had HA in MRI1 and 7 (70\%; Figure 3).

\section{Sporadic mesial temporal lobe epilepsy}

Twenty-seven patients (82\%) had HA in the MRI1. Two patients with normal first MRI presented HA at the second scan (29/33 patients, $88 \%$ ). Group analysis demonstrated a significant difference between the volumes of patients and controls for both the first and second MRI ( $t$-test MR1: right hippocampus, $p=0.02$; left hippocampus, $p<0.000$; AI, $p<0.0001$. MR2: right hippocampus, $p=0.004$; left hippocampus, $p<0.001$; AI, $p<0.001$; Figure 1).

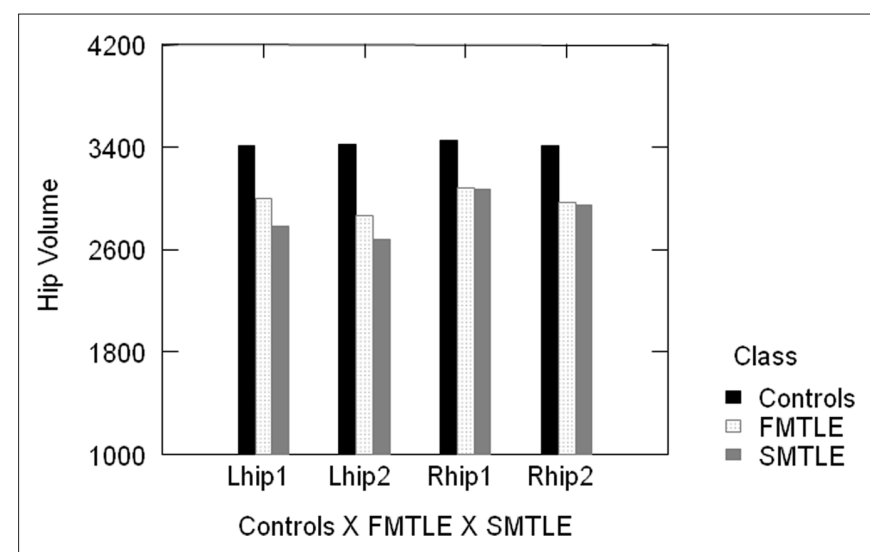

FIGURE 1 | Group analysis demonstrated significant difference of hippocampal volumes of controls and patients (FMTLE and SMTLE) for both the first and second MRI. Lhip1, left hippocampus in the first MRI; Lhip2, left hippocampus in the second MRI; Rhip1, right hippocampus in the first MRI; Rhip2, right hippocampus in the second MRI. 


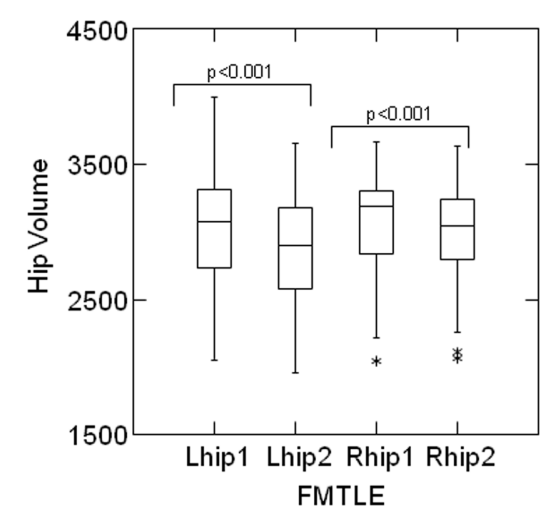

FIGURE 2 | Box and whisker plots: the bottom and top of the box represents the 25th and 75th percentile (the lower and upper quartiles, respectively), and the band near the middle of the box is the 50th percentile (the median). The "*" means outliers. Paired t-test comparing MRI1 and MRI2 showed a

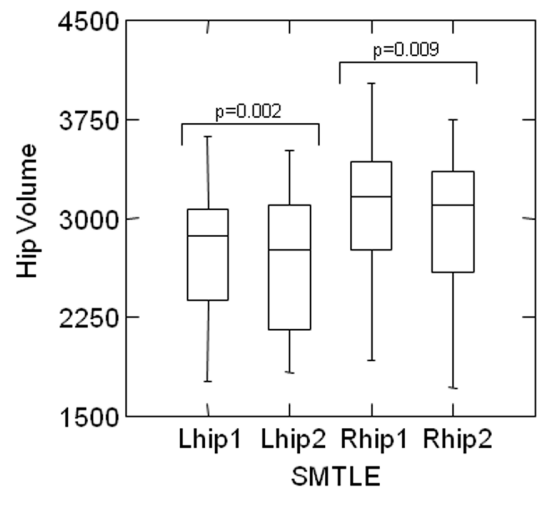

significant reduction of the right and left hippocampal volumes during the follow-up period in FMTLE and SMTLE groups. Lhip1, left hippocampus in the first MRI; Lhip2, left hippocampus in the second MRI; Rhip1, right hippocampus in the first MRI; Rhip2, right hippocampus in the second MRI.
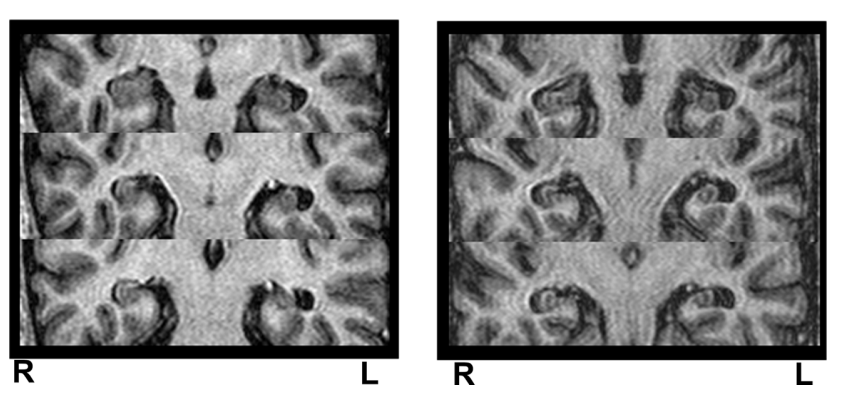

FIGURE 3 | Example of MRI acquired at time zero (MRI 1) and after 20 months (MRI 2), in a patient with FMTLE. Hippocampal volumetry demonstrated left hippocampal atrophy at MRI 1 (left Z-score $=-2.33$ ) and bilateral hippocampal atrophy at MRI 2 (left Z-score $=-3.63$ and right $Z$-score $=-2.81$ ), indicating progression of left hippocampal atrophy and the development of right hippocampal atrophy overtime. $R$, right side; $L$, left side.

Paired $t$-test comparing MRI1 and MRI2 showed a significant reduction of the right $(p=0.009)$ and left $(p=0.0025)$ hippocampal volumes during the follow-up period (Figure 2).

We also subdivided the SMTLE patients in benign [4 (12\%) individuals; 2 (50\%) with HA in MR1 and 3 (75\%) with HA in MRI2] and refractory [29 individuals; 25 (86\%) with HA in MR1 and $26(90 \%)$ with HA in MRI2]. However, the number of patients in the benign group was too small for statistical analysis.

Although the mean time between the MRIs in the FMTLE group was twice the time of the SMTLE group, there was no difference of degree of volume reduction in MRI2 among the two groups (FMTLE or SMTLE; $t$-test, right hippocampus: $p=0.886$, and left hippocampus: $p=0.598$ ).

\section{Control group}

Paired $t$-test comparing MRI1 and MRI2 failed to demonstrate a significant reduction of the right $(p=0.259)$ or left $(p=0.888)$ hippocampal volumes during the follow-up period in the control group (Figure 1).

\section{DISCUSSION}

As demonstrated by previous studies, we observed that FMTLE patients commonly have HA detected by post-processing MRI techniques (57-66\%; Kobayashi et al., 2001, 2003), although this is less frequent than observed in SMTLE (82-88\%; Watson et al., 1997; Fuerst et al., 2003; Cendes et al., 2005). What we were able to demonstrate for the first time is that, like in SMTLE (Watson et al., 1997; Pitkänen et al., 2002; Cendes, 2005; Bonilha et al., 2006; Bernhardt et al., 2009; Coan et al., 2009), FMTLE patients have hippocampal volume reduction over time, independently of seizure frequency.

Familial mesial temporal lobe epilepsy is considered a benign form of epilepsy, although some patients present with medically refractory seizures and require surgical treatment (Berkovic et al., 1996; Kobayashi et al., 2001, 2002, 2003). In the present study the majority of patients had a benign form of FMTLE (71\%) as expected, while the majority of SMTLE individuals were refractory (88\%). Previous clinical (Cendes, 2005; Bonilha et al., 2006; Coan et al., 2009) and experimental (Bonilha et al., 2006) studies of refractory MTLE have shown progressive atrophy of mesial temporal lobe structures and also of neocortical structures (Bonilha et al., 2006; Bernhardt et al., 2009; Coan et al., 2009) over time and some also correlated the seizure frequency with the progression of damage (Kalviainen et al., 1998; Briellmann et al., 2002; Fuerst et al., 2003; Cendes, 2005; Cendes et al., 2005). However, there are no studies showing unequivocal evidence of progressive damage in patients with benign forms of epilepsy, such as FMTLE.

We made an option, in this study, to consider as benign those patients with up to three complex partial seizures per year or only simple partial seizures, although this definition is subjective (Kobayashi et al., 2001). However, for better understanding of MTLE some classification must be used to try to clarify these gaps (Labate et al., 2011). We hypothesized that seizure frequency has influence on the evolution of the disease, as demonstrated by previous clinical, experimental, neuroimaging, and neuropsychological studies (Kalviainen et al., 1998; Pitkänen et al., 2002; Fuerst et al., 2003; Cendes, 2005; Bonilha et al., 2006; Bernhardt et al., 2009; Coan et al., 2009). Although our classification is arbitrary, 
it allowed the separation of two extremes of seizure control and to evaluate their neuroimaging evolution. We may also add the fact that those patients who presented only simple partial seizures during the follow-up and were classified here as benign, had typical psychic or autonomic auras of MTLE which were not disabling.

Although FMTLE is described as a benign condition, in our group a significant number of patients had frequent seizures. With this classification (benign $\mathrm{X}$ refractory) our main purpose was to clarify if refractory FMTLE had the same evolution as refractory SMTLE. Indeed, when we analyzed the progression of HA over time in the two groups (benign FMTLE and refractory FMTLE) both had equivalent hippocampal volume reduction. Unfortunately, we were not able to determine if the benign SMTLE patients had significant progression of HA, once the number of individuals in this group was too small.

It would be important to analyze these individuals in smaller groups concerning, for instance, sex, age of epilepsy onset or duration of epilepsy, history, and type of initial precipitating injury. However, the number of patients in these subgroups would be too small for the statistical analysis. We strongly believe that further studies, with a larger number of patients and more detailed MRI evaluation, as the measure of other mesial temporal structures would be interesting.

The fact that not only the FMTLE patients had a significant loss of hippocampal volume along the time, but also when we subdivided this group, the benign FMTLE individuals also had significant progression of HA is an important finding. We may speculate that the hippocampal volume reduction over time is not clinically significant to seizure control in FMTLE patients, since the group of benign individuals continued to be well controlled (most of them were seizure-free with or without medication) during this period.

Our results also demonstrated that although the mean time between the MRIs in the FMTLE group was twice the time of the SMTLE group, the degree of volume reduction was not different between the two groups. If the progression of HA was similar in both groups, we would expect to find more pronounced volume reduction in FMTLE. We may hypothesize that this slower hippocampal reduction in FMTLE happened because of the difference of refractory patients in both groups ( $88 \%$ of SMTLE vs $29 \%$ of FMTLE), although the benign FMTLE also had significant volume reduction. Most probably the mechanisms related to the progression of damage in these two groups, SMTLE and FMTLE, are diverse with an important contribution of the genetic characteristics in FMTLE. This is corroborated by a recent paper from our group emphasizing the stronger environmental influence in patients with MTLE without a family history that could influence the more widespread brain structural abnormalities and worse IQ performance found in SMTLE patients (Yasuda et al., 2010).

We acknowledge that the significant difference in the interval of the MRIs between FMTLE and SMTLE is a possible limitation of our study; however, it propitiated an interesting result. The difference in the interval of MRIs can be explained mainly because the patients with refractory seizures were on the waiting list for surgical treatment and it was not ethical to wait too long to do a second MRI before surgery. On the other hand, as the majority of FMTLE individuals were benign, they could have a second MRI after a longer interval. Additionally, we had the prior hypothesis that the FMTLE patients would have no or very mild progression, since most of them had a "benign" clinical course (remission, seizure control, or rare seizures). If they had two MRIs with a short interval and we did not find a significant hippocampal volume difference we would be left with the question that it could have been due to the short interval or low seizure frequency in this group.

The fact that seizures are responsible for additional damage in patients with epilepsy is controversial (Cendes, 2005). While some neuroimaging studies describe a significant relation of seizure frequency and cerebral volume reduction of patients with refractory epilepsy (Bonilha et al., 2006; Bernhardt et al., 2009; Coan et al., 2009), other studies did not agree, including a post-mortem study that did not find a relationship between seizure frequency and hippocampal neuronal loss (Thom et al., 2005). In contrast, a recent study demonstrated that MTLE patients with refractory seizures had more significant and diffuse gray matter damage than those that were seizure-free or had remitting-relapsing evolution (Bilevicius et al., 2010). This difference maybe predominantly related to the heterogeneity of patients included in each study and the different techniques used to address the progression of atrophy.

Actually, it is important to address that MTLE is not a single disease (Cendes et al., 2005; Berg, 2008) but a clinical and electroencephalographic syndrome. The diverse prognostic and evolution seen on different MTLE patients may be related to different etiological factors or diverse initial precipitating injuries. Studies with a larger number and more homogeneous individuals are necessary to clarify these questions.

It is most likely that seizure frequency causes further hippocampal damage and consequently volume reduction in only some types of epilepsies or epileptic patients. In addition, seizure types and duration of habitual seizures may influence neuronal damage differently according to the basic etiological mechanism implicit on each different individual. When we focus on MTLE we are surely evaluating epilepsies with diverse etiologies and the causes of neuronal damage will certainly be different, and may not necessarily be directly related to the seizures per se, but to the underlying mechanisms of seizure generation. Therefore, this could explain the fact that a phenomenologically similar seizure type (with similar frequency) in two different patients may cause more damage in one than in the other.

In this specific study, we did not aim to analyze other MRI features of HS, such as T2 hyperintense signal, loss of internal architecture, or abnormal hippocampal axis. This more subtle abnormalities would require a bigger number of patients to show significant results. Indeed, the most reliable MRI finding of HS is the presence of HA combined with hyperintense T2 signal. If we analyze any of the MRI findings isolated, HA is the most reliable MRI feature of HS. Hyperintense T2 signal can be present in some patients without atrophy, but in a very small proportion of patients. In addition, when we take any of these two findings isolated, there is a higher chance of false positives for hyperintense T2 signal than for atrophy (Cendes and Cascino, 2010; Labate et al., 2010). 
In summary, FMTLE patients have hippocampal volume reduction over time independently of seizure frequency although this progression of damage seems to be slower than what occurs in SMTLE.

\section{REFERENCES}

Berg, A. T. (2008). The natural history of mesial temporal lobe epilepsy. Curr. Opin. Neurol. 21, 173-178.

Berkovic, S. F., Mcintosh, A., Howell, R. A., Mitchell, A., Sheffield, L. J., and Hopper, J. L. (1996). Familial temporal lobe epilepsy- a common disorder identified in twins. Ann. Neurol. 40, 227-235.

Bernhardt, B. C., Worsley, K. J., Kim, H., Evans, A. C., Bernasconi, A., and Bernasconi, N. (2009). Longitudinal and cross-sectional analysis of atrophy in pharmacoresistant temporal lobe epilepsy. Neurology 72, 1747-1754.

Bilevicius, E., Yasuda, C. L., Silva, M. S., Guerreiro, C.A., Lopes-Cendes, I., and Cendes, F. (2010). Antiepileptic drug response in temporal lobe epilepsy: a clinical and MRI morphometry study. Neurology 75, 1695-1701.

Bonilha, L., Rorden, C., Appenzeller, S., Coan, A. C., Cendes, F., and Li, L. M. (2006). Gray matter atrophy associated with duration of temporal lobe epilepsy. Neuroimage 32, 1070-1079.

Briellmann, R. S., Berkovic, S. F., Syngeniotis, A., King, M. A., and Jackson, G. D. (2002). Seizureassociated hippocampal volume loss: a longitudinal magnetic resonance study of temporal lobe epilepsy. Ann. Neurol. 51, 641-644.

Bruton, C. J. (1988). The Neuropathology of Temporal Lobe Epilepsy. New York: Oxford University Press.

Cascino, G. D., Jack, C. R. Jr., Parisi, J. E., Sharbrough, F. W., Hirschorn, K. A., Meyer, F. B., Marsh, W. R., and O’Brien, P. C. (1991). Magnetic resonance imaging-based volume studies in temporal lobe epilepsy: pathological correlations. Ann. Neurol. 30, 31-36.

Cendes, F. (2005). Progressive hippocampal and extrahippocampal atrophy in drug resistant epilepsy. Curr. Opin. Neurol. 18, 173-177.
Cendes, F., Andermann, F., Gloor, P., Evans, A., Jones-Gotman, M., Watson, C., Melanson, D., Olivier, A., Peters, T., Lopes-Cendes, I., and Leroux, G. (1993). MRI volumetric measurements of amygdala and hippocampus in temporal lobe epilepsy. Neurology $43,719-725$.

Cendes, F., and Cascino, G. D. (2010).MRI signs of hippocampal sclerosis seen in healthy volunteers: what is the clinical relevance? Neurology 74, 534-535.

Cendes, F., Kahane, P., Brodie, M., and Andermann, F. (2005). "The mesiotemporal lobe epilepsy syndrome," in Epileptic Syndromes in Infancy, Childhood and Adolescence, 4th Edn, eds J. Roger, M. Buerau, C. Dravet, P. Genton, C. A. Tassinari, and P. Wolf (Montrouge: John Libbey Eurotext), 555-575.

Coan,A. C., Appenzeller, S., Bonilha, L., Li, L. M., and Cendes, F. (2009). Seizure frequency and lateralization affect progression of atrophy in temporal lobe epilepsy. Neurology 73, 834-842.

Fuerst, D., Shah, J., Shah, A., and Watson, C. (2003). Hippocampal sclerosis is a progressive disorder: a longitudinal volumetric MRI study. Ann. Neurol. 53, 413-416.

Gastaut, H., Gastaut, J. L., Gonçalves e Silva, G. E., and Fernandez Sanchez, G. R. (1975). Relative frequency of different types of epilepsy: a study employing the classification of the international league against epilepsy. Epilepsia 16, 457-461.

Jackson, G. D., Connely, A., Duncan, J. S., Grünewald, R. A., and Gadian, D. G. (1993). Detection of hippocampal pathology in intractable partial epilepsy: increased sensitivity with quantitative magnetic resonance T2 relaxometry. Neurology 43, 1793-1799.

Kälviäinen, R., Salmenperä, T., Partanen, K., Vainio, P., Riekkinen, P., and

Up to now, there is important evidence on literature that epilepsy is a progressive disorder with variable severity. It is important to define in which particular group of patients it happens and what the specific causes of this progression are.

Pitkänen, A. (1998). Recurrent seizures may cause hippocampal damage in temporal lobe epilepsy. Neurology 50, 1377-1382.

Kobayashi, E., D’Agostinho, M. D., LopesCendes, I., Berkovic, S. F., Li, M. L. Andermann, E., Andermann, F., and Cendes, F. (2003). Hippocampal atrophy and T2-weighted signal chances in familial mesial temporal lobe epilepsy. Neurology 60, 405-409.

Kobayashi, E., Li, M. L., Lopes-Cendes, I., and Cendes, F. (2002). Magnetic resonance imaging evidence of hippocampal sclerosis in asymptomatic, first-degree relatives of patients with familial mesial temporal lobe epilepsy. Arch. Neurol. 59, 1891-1894.

Kobayashi,E., Lopes-Cendes, I., Guerreiro, C. A., Sousa, S. C., Guerreiro, M. M. and Cendes, F. (2001). Seizure outcome and hippocampal atrophy in familial mesial temporal lobe epilepsy. Neurology 56, 166-172.

Labate, A., Gambardella, A., Aguglia, U., Condino, F., Ventura, P., Lanza, P., and Quattrone, A. (2010). Temporal lobe abnormalities on brain MRI in healthy volunteers: a prospective case-control study. Neurology 74, 553-557.

Labate, A., Gambardella, A., Andermann, E., Aguglia, U., Cendes, F., Berkovic, S. F., and Andermann, F. (2011). Benign mesial temporal lobe epilepsy. Nat. Rev. Neurol. [Epub ahead of print].

Pitkänen, A., Nissinen, J., Nairismägi, J., Lukasiuk, K., Gröhn, O. H., Miettinen, R., and Kauppinen, R. (2002). Progression of neuronal damage after status epilepticus and during spontaneous seizures in a rat model of temporal lobe epilepsy. Prog. Brain Res. 135, 67-83.

Thom, M., Zhou, J., Martinian, L., and Sisodiya, S. (2005). Quantitative postmortem study of the hippocampus in chronic epilepsy: seizures do not inevi- tably cause neuronal loss. Brain 128, 1344-1357.

Watson, C., Jack, C. R. Jr., and Cendes, F. (1997). Volumetric magnetic resonance imaging. Clinical applications and contributions to the understanding of temporal lobe epilepsy. Arch. Neurol. 54, 1521-1531.

Yasuda, C. L., Morita, M. E., Alessio, A., Pereira, A. R., Balthazar, M. L., Saúde, A. V., Costa,A. L., Costa,A. L., Cardoso, T. A., Betting, L. E., Guerreiro, C. A., Damasceno, B. P., Lopes-Cendes, I., Tedeschi, H., de Oliveira, E., and Cendes, F. (2010). Relationship between environmental factors and gray matter atrophy in refractory MTLE. Neurology 74, 1062-1068.

Conflict of Interest Statement: The authors declare that the research was conducted in the absence of any commercial or financial relationships that could be construed as a potential conflict of interest.

Received: 12 October 2010; accepted: 30 January 2011; published online: 14 February 2011.

Citation: Conz L, Morita ME, Coan AC, Kobayashi E, Yasuda CL, Pereira AR, Lopes-Cendes I and Cendes F (2011) Longitudinal MRI volumetric evaluation in patients with familial mesial temporal lobe epilepsy. Front. Neur. 2:5. doi: 10.3389/ fneur.2011.00005

This article was submitted to Frontiers in Epilepsy, a specialty of Frontiers in Neurology.

Copyright (C) 2011 Conz, Morita, Coan, Kobayashi, Yasuda, Pereira, Lopes-Cendes and Cendes. This is an open-access article subject to an exclusive license agreement between the authors and Frontiers Media $S A$, which permits unrestricted use, distribution, and reproduction in any medium, provided the original authors and source are credited. 